\author{
Cadernos de \\ ESTUDOS LINGḯlistICOS - (54.1), Campinas, Jan./Jun. 2012
}

\title{
SÉRIES TEMPORAIS DE PAUSAS E DE HESITAÇÕES NA FALA ESPONTÂNEA*
}

\author{
SANDRA MERLO** \\ PLÍNIO ALMEIDA BARBOSA**
}

\begin{abstract}
RESUMO
O objetivo deste estudo é examinar a dinâmica de longo prazo de pausas e hesitações na fala espontânea. Foram analisados 20 textos falados, produzidos por adultos sem distúrbios de comunicação. Os dados foram transcritos e segmentados de acordo com os fenômenos de interesse. Posteriormente, os dados foram transformados em sequências numéricas de "zeros" e "uns" e amostrados a cada 200 ms para a geração das séries temporais. Foram utilizados procedimentos estatísticos específicos de séries temporais para análise dos dados. Os resultados apontaram que pausas e hesitações ocorreram de forma estacionária do início ao fim dos textos falados, ou seja, não se acumularam no início, no meio ou no final dos textos. Pausas e hesitações se distribuíram de forma cíclica e periódica ao longo do tempo: os ciclos de pausa apresentaram média de $9 \mathrm{~s}$ e os de hesitação, média de $13 \mathrm{~s}$. Pausas e hesitações atuaram em conjunto para a manutenção da fluência, tendo em vista que mudanças de estado em uma série imediatamente geraram mudanças de estado na outra série temporal. Houve poucas ocorrências de ciclos sincronizados de pausas e hesitações e, quando isso ocorreu, os fenômenos geralmente estavam em oposição de fase, ou seja, regiões com maior concentração de pausas ocorreram com regiões de menor concentração de hesitações. Portanto, a estacionaridade e a periodicidade podem ser consideradas modos robustos e estáveis de organização temporal das pausas e hesitações na fala espontânea.
\end{abstract}

Palavras-Chave: Fluência; estacionaridade; ciclos periódicos.

\begin{abstract}
The aim of this research is to examine long-term dynamics of pauses and hesitations in spontaneous speech. Twenty spoken texts, produced by adults without communication disorders, were analyzed. The data were transcribed and segmented according to the phenomena under consideration. Afterwards, data were transformed in sequences of "zeros" and "ones" and sampled at a $200 \mathrm{~ms}$ rate for being analyzed as time series. Statistical procedures specific to time series analysis were employed to study the data. Results indicate that pauses and hesitations occur in a stationary fashion from the beginning to the end of spoken texts, i. e. they did not accumulate at the beginning, in the middle or at the end of the texts. Pauses and hesitations occur cyclically and periodically through time: pause cycles have a mean
\end{abstract}

\footnotetext{
*. Agradecemos aos sujeitos que participaram desta pesquisa. Também agradecemos o financiamento concedido pelo CNPq (processo: 140281/2007-0).

**. UNICAMP/IEL - Departamento de Linguística, Grupo de Estudos de Prosódia da Fala. Campinas (SP), Brasil. e-mail: sgmerlo@gmail.com

***. UNICAMP/IEL - Departamento de Linguística, Grupo de Estudos de Prosódia da Fala. Campinas (SP), Brasil. e:mail: pabarbosa.unicampbr@gmail.com
} 
MERLO e ALMEIDA BARBOSA - Séries temporais de pausas...

duration of $9 \mathrm{~s}$ and hesitation cycles have a mean duration of $13 \mathrm{~s}$. Pauses and hesitations work together to maintain speech fluency, considering that changes in one series caused immediate changes in the other series. There were a few instances of synchronized cycles of pauses and hesitations in the data and, when this happened, phenomena usually were in phase opposition, i. e. regions with more pauses relate with regions with less hesitations. Therefore, stationarity and periodicity can be considered as robust and stable manners of temporal organization of pauses and hesitations in spontaneous speech.

Keywords: Fluency; stationarity; periodic cycles.

\section{INTRODUÇÃO}

Este trabalho se debruça sobre dois fenômenos genuinamente falados e cruciais para a fluência: as pausas silenciosas fluentes e as hesitações. Nosso interesse reside especificamente na maneira como esses fenômenos se comportam ao longo do tempo na fala espontânea.

As pausas silenciosas fluentes (doravante, pausas) são silêncios que ocorrem durante a fala, estando localizadas preferencialmente em constituintes maiores e apresentando ampla variação de pitch antes e depois da pausa (Campione \& Véronis, 2004). A função textual das pausas é segmentar a produção falada em unidades semânticas, sintáticas e/ou prosódicas (Barbosa, 2006; Campione \& Véronis, 2004). Sua função psicolinguística relaciona-se com o processamento da informação ${ }^{1}$ que será verbalizada (Kircher et al., 2004).

O termo "hesitação" engloba uma série de marcas linguísticas que o falante utiliza quando fala. A função textual da hesitação é desacelerar o texto falado (Souza e Silva \& Koch, 2002). Sua função psicolinguística é fornecer tempo adicional para o falante decidir sobre a verbalização da informação (Kircher et al., 2004; Koch \& Souza e Silva, 1996; Marcuschi, 2006). A desaceleração da produção pode dar a impressão de perda de fluência, mas, na verdade, a ocorrência da hesitação atua para manter a fluência, na medida em que auxilia o falante a produzir enunciados mais precisos (O'Connell \& Kowal, 2004). O grau de familiaridade com o enunciado parece ser o principal motivador da hesitação: enunciados pouco familiares e pouco automatizados aumentam o número de hesitações, ao passo que enunciados mais familiares e mais automatizados tendem a apresentar poucas hesitações (Scarpa, 1995, 2006; Schachter et al., 1991).

Tendo em vista que pausas e hesitações ocorrem ao longo de toda a extensão dos textos falados, consideramos apropriado estudar a dinâmica temporal de longo prazo desses fenômenos. Nosso interesse está em verificar (a) se a distribuição de pausas e hesitações é estacionária, ou seja, se ocorrem ou não aumentos ou diminuições significativas na manifestação desses fenômenos ao longo do texto falado e (b) se pausas e hesitações se distribuem cíclica e periodicamente em textos falados espontâneos, ou seja, se regiões de maior concentração se alternam regularmente com regiões de menor concentração de pausas e hesitações.

1. Por "processamento da informação", nos referimos às chamadas funções executivas (Kircher et al., 2004), como por exemplo, recuperação de informações da memória de longo prazo, escolha de estratégias para organizá-las e monitoramento do desempenho. 
Diversos estudos demonstraram que pausas e hesitações não estão distribuídas aleatoriamente ao longo do tempo durante a fala espontânea e o tópico textual tem se mostrado uma variável linguística relevante neste processo. Jaffe et al. (1964) demonstraram que o falante controla a produção de pausa silenciosa (fluente ou hesitativa) em pelo menos duas escalas diferentes de tempo: em curtíssimo prazo (a cada $333 \mathrm{~ms}$ ) e em curto prazo (a cada 4 ou 5 s). Warner (1979) mostrou que o controle da produção de pausas silenciosas (fluentes e hesitativas) também pode ocorrer em médio prazo, com ciclos periódicos de 3 a 6 minutos, e que os falantes sincronizam seus ciclos durante conversações. Greene \& Cappella (1986) mostraram que também pode haver um controle de longo prazo sobre a ocorrência de pausas silenciosas (fluentes e hesitativas), tendo em vista que o conhecimento prévio da sequência de subtópicos de um texto diminui a ocorrência de pausas ao longo de todo o texto falado. Roberts \& Kirsner (2000) também encontraram um controle de médio prazo sobre a produção de pausas silenciosas (fluentes e hesitativas) e pausas preenchidas na fala espontânea, com ciclos periódicos na ordem de $17 \mathrm{~s}$, sendo que a ocorrência de pausas aumentava nas regiões de mudança de tópico.

No estudo atual, iremos comparar a dinâmica temporal de longo prazo de pausas e hesitações em textos produzidas por falantes adultos do português brasileiro. Levando em consideração que estudos anteriores evidenciaram a relevância do tópico textual, optamos por avaliar o papel da macroestrutura textual e da temática autobiográfica para a manifestação de pausas e hesitações.

A macroestrutura fornece um esquema para a organização das informações no texto. Os tipos textuais escolhidos (descrição de estado e narrativa) são bastante diferentes entre si (Adam \& Revaz, 1997). As descrições de estado são textos em que não há sequência prévia das informações, ou seja, o falante pode sequenciar as informações da maneira que achar mais apropriado: ele pode seguir uma organização espacial (da esquerda para a direita, por exemplo) ou pode optar por organizar as informações segundo algum princípio funcional (Levelt, 1989). Nas narrativas, por outro lado, a ordem das informações costuma estar relativamente pré-determinada, sendo a ordenação cronológica o princípio canônico. A descrição é a base da narrativa, tendo em vista que o falante aprende a descrever antes de aprender a narrar.

Temos hipóteses inversas para os ciclos de pausas e hesitações em relação ao tipo textual:

a. A inexistência, nas descrições de estado, de uma ordem canônica para sequenciar as informações fará com que os falantes precisem decidir ativamente sobre este aspecto (Adam \& Revaz, 1997). Não sendo um processamento automatizado, os falantes precisarão monitorá-lo com mais atenção. Neste sentido, são esperados ciclos de pausa com períodos menores nas descrições de estado, evidenciando o monitoramento mais de curto prazo da produção falada. Nas narrativas, por outro lado, existe uma ordem canônica previamente aprendida. Assim, são esperados ciclos de pausa com períodos maiores nas narrativas, evidenciando o monitoramento de longo prazo neste tipo textual. 
MERLO e ALMEIDA BARBOSA - Séries temporais de pausas...

b. Visto que o falante aprende a descrever antes de aprender a narrar (Adam \& Revaz, 1997), os enunciados descritivos são mais familiares do que os narrativos. Neste sentido, são esperados ciclos de hesitação com períodos maiores nas descrições, evidenciando o monitoramento mais de longo prazo deste tipo textual. Por outro lado, os enunciados narrativos, sendo menos familiares do que os descritivos, necessitam de maior monitoramento. Assim, são esperados ciclos de hesitação com períodos menores nas narrativas, evidenciando o monitoramento mais de curto prazo da produção.

O conhecimento adquirido pode ou não ser autobiográfico (Dritschel et al., 1992). Conhecimentos autobiográficos dizem respeito às vivências individuais, podendo ser aprendidos em experiências únicas (episódios) ou repetidas. Já os conhecimentos não-autobiográficos são compartilhados socialmente, generalizados e descontextualizados, sendo mais comumente aprendidos em experiências repetidas. Os conhecimentos não-autobiográficos são mais flexíveis do que os autobiográficos, porque podem ser mais facilmente aplicados a novos contextos. Os conhecimentos não-autobiográficos, por geralmente serem aprendidos com experiências repetidas, são mais facilmente recordados do que os autobiográficos (Dritschel et al., 1992).

Temos hipóteses inversas para os ciclos de pausas e hesitações em relação à temática autobiográfica:

a. A utilização de conhecimentos não-autobiográficos em uma situação concreta, exige que o falante decida ativamente sobre a adaptação daquele conhecimento àquela situação em particular. Assim, é preciso haver um monitoramento mais de curto prazo durante a aplicação de conhecimentos nãoautobiográficos. Por isso, esperamos ciclos de pausa com períodos menores nos textos não-autobiográficos. $\mathrm{O}$ mesmo não acontece com a utilização de conhecimentos autobiográficos, os quais podem ser apenas relatados. Assim, esperamos ciclos de pausa com períodos maiores nos textos autobiográficos, evidenciando o monitoramento mais de longo prazo.

b. Os conhecimentos não-autobiográficos são mais facilmente recordados e verbalizados do que os autobiográficos. Desta forma, sua produção é mais automatizada, não necessitando de monitoramento tão intenso. Por isso, esperamos ciclos de hesitação com períodos maiores nos textos não-autobiográficos. Por outro lado, a verbalização de conhecimentos autobiográficos não é tão automatizada, necessitando de maior monitoramento. Por isso, esperamos ciclos de hesitação com períodos menores nos textos autobiográficos.

Há a sugestão de que pausas e hesitações ocorrem em maior concentração no início dos textos falados devido ao processo de decisão, por parte do falante, de qual será o foco do texto (Marcuschi, 2006). Por outro lado, Roberts \& Kirsner (2000) e Warner (1979) não referem a utilização de métodos para retirar tendências não-estacionárias dos dados, o que indica que eles eram estacionários. Nossa hipótese é que a necessidade de processar e verbalizar informações ocorre ininterruptamente do começo ao fim do texto falado, não havendo uma região preferencial de maior esforço cognitivo. 


\section{MÉTODO}

Participaram deste estudo cinco sujeitos do sexo masculino, entre 20 e 34 anos, com nível superior de escolaridade e falantes nativos do português brasileiro. Nenhum dos sujeitos relatou queixa (atual ou pregressa) de distúrbio de audição, fala, leitura ou escrita.

As gravações foram realizadas em cabine acústica. Cada sujeito produziu quatro textos falados: descrição da figura de um quarto (Moraes, 2003), descrição de seu próprio quarto, narração de uma estória em quadrinhos sem texto (Aragonés, 1999) e narração de uma estória engraçada que viveu. Os textos foram analisados conforme o tipo textual (descrição de estado ou narrativa) e quanto à característica autobiográfica (textos sem apoio pictórico: autobiográficos, textos com apoio pictórico: não-autobiográficos).

Todas os textos falados foram transcritos com o auxílio das camadas de anotação do software Praat (Boersma \& Weenink, 1992-2010). Para a segmentação dos períodos de pausa e hesitação, foram utilizados os seguintes critérios:

- Pausa silenciosa fluente: período de silêncio, localizado entre sintagmas, havendo mudança no movimento da curva de F0 antes e depois da pausa (Campione \& Véronis, 2004).

- Hesitação: momentos de desaceleração em qualquer nível linguístico (fonético, fonológico, morfológico, sintático ou semântico), caracterizados pelas seguintes manisfestações (Campione \& Véronis, 2004; Marcuschi, 2006; Wingate, 1987):

- Pausa silenciosa hesitativa: período de silêncio, localizado no interior de sintagmas, não havendo mudança no movimento da curva de F0 antes e depois da pausa.

- Pausa preenchida: sons de preenchimento, que, em português brasileiro, costumam ser transcritos ortograficamente como "éh", "ãh", "ah", "mm".

- Repetição hesitativa: ocorrências sucessivas de um mesmo trecho, sem função de ênfase. As repetições podem ser de parte de palavra (fone ou sílaba) ou de palavras (isoladas ou em sequência).

- Alongamento hesitativo: aumento na duração de um fone, sem função de ênfase, em início ou final de palavra.

- Falso início: construção problemática, que pode ser abandonada ou corrigida.

- Bloqueio: posição articulatória fixa, que ocasiona suspensão temporária do enunciado.

A segmentação também considerou a unidade de vogal a vogal, que é uma sílaba fonética. Todos os trechos de segmentação iniciaram no padrão formântico de onset de uma vogal e terminaram no padrão formântico de onset da próxima vogal (Barbosa, 2006). No caso das pausas silenciosas, portanto, o trecho segmentado nunca foi apenas da pausa, mas da vogal que a precedia e da própria pausa silenciosa. 
MERLO e ALMEIDA BARBOSA - Séries temporais de pausas...

Os períodos de pausa e de hesitação receberam rótulos numéricos em suas respectivas camadas de segmentação no software Praat: períodos de ocorrência de pausa ou hesitação receberam o número " 0 ", enquanto períodos sem a ocorrência de pausa ou hesitação receberam o número " 1 ". Portanto, cada texto falado foi transformado em duas sequências numéricas (uma de pausa e não-pausa e outra de hesitação e não-hesitação). Os textos foram posteriormente amostrados a cada 200 ms para a geração das séries temporais de pausa e hesitação.

Para análise das séries temporais, foram utilizados procedimentos gráficos descritivos e inferenciais (Chatfield, 2004; Morettin \& Toloi, 2004).

- Em relação à análise univariada no domínio da frequência, foi utilizada análise espectral para estimar a amplitude de cada frequência do espectro, com os resultados sendo representados graficamente pelo periodograma. A ordenada de $0 \mathrm{~Hz}$ do periodograma foi inspecionada para verificação ou não da estacionaridade da série temporal, sendo a ausência de pico em $0 \mathrm{~Hz}$ indicativa de que a série é estacionária. O teste de Fisher foi utilizado para cálculo de significância estatística das ordenadas do periodograma (nível de significância $\alpha=0,05$ ). Os períodos (em segundos) dos ciclos de pausa e de hesitação foram comparados com o teste t.

- Em relação à análise bivariada, foi utilizado o coeficiente de correlação cruzada para detecção de relações de precedência ou defasagem entre as séries temporais de pausa e hesitação (Warner, 1998). Também foi utilizada análise espectral cruzada quando as séries temporais de pausa e hesitação apresentaram ciclos de mesmo período, com o intuito de verificar se o ciclo de uma série temporal estava sincronizado com o ciclo da outra série (Warner, 1998). Seguindo recomendações da literatura (Chatfield, 2004; Warner, 1998), os resultados relativos à correlação ao quadrado (coerência) e à relação de fase foram utilizados para inferir sobre a presença de ciclos sincronizados entre as séries de pausa e hesitação. A coerência será considerada alta quando for igual ou superior a $50 \%$.

\section{RESULTADOS E DISCUSSÃO}

Todos os 20 textos falados apresentaram pausas e hesitações. A duração dos textos variou entre 21 e 222 s (com média de 70 s). Ou seja, nosso corpus é constituído por textos curtos e as interpretações que serão feitas levarão em conta este fato.

Todos os textos apresentaram comportamento estacionário de pausas e hesitações, isto é, não houve diminuição ou aumento significativo desses fenômenos ao longo do tempo (Figuras 1 e 2). Alguns textos do nosso corpus apresentaram acúmulo de pausas ou hesitações em alguma região do texto: por exemplo, na Figura 3, aparentemente há um acúmulo de pausas na segunda metade do texto e, na Figura 4, aparentemente há um acúmulo de hesitações na região 
central do texto. Entretanto, os resultados indicaram que esses acúmulos ocorrem de forma aleatória e não-significativa. Há a sugestão de que pausas e hesitações ocorrem em maior concentração no início dos textos falados devido ao processo de decisão sobre o foco informativo do texto (Marcuschi, 2006). Por um lado, há a possibilidade de que o experimento não tenha propiciado a busca do foco textual, mas, por outro, também há a possibilidade de que seja enganosa a impressão de que pausas e hesitações se acumulam no início das produções. Então, concluímos que a necessidade de processar e verbalizar informações ocorre ininterruptamente do começo ao fim do texto falado, não havendo uma região preferencial de maior esforço cognitivo.

Figura 1: Série temporal de pausas silenciosas fluentes. Texto com $37 \mathrm{~s}$ de duração, produzido pelo sujeito 5 na descrição autobiográfica. $\mathrm{Na}$ ordenada, o valor " 0 " indica a ocorrência de "pausa silenciosa fluente", enquanto o valor "1" indica o seu contrário. Na abscissa, cada unidade equivale a $200 \mathrm{~ms}$.

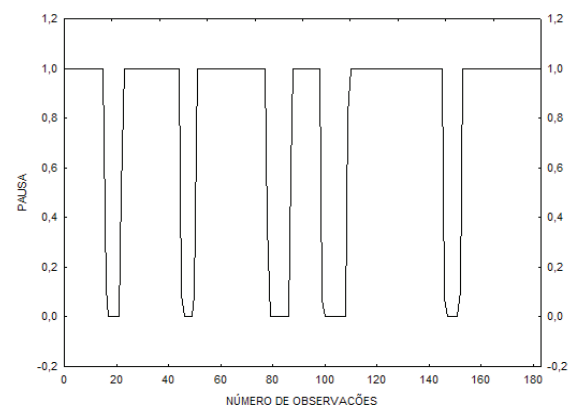

Figura 3: Série temporal de pausas silenciosas fluentes. Texto com $21 \mathrm{~s}$ de duração, produzido pelo sujeito 3 na narrativa de figura. $\mathrm{Na}$ ordenada, o valor " 0 " indica a ocorrência de "pausa silenciosa fluente", enquanto o valor " 1 " indica o seu contrário. $\mathrm{Na}$ abscissa, cada unidade equivale a $200 \mathrm{~ms}$.

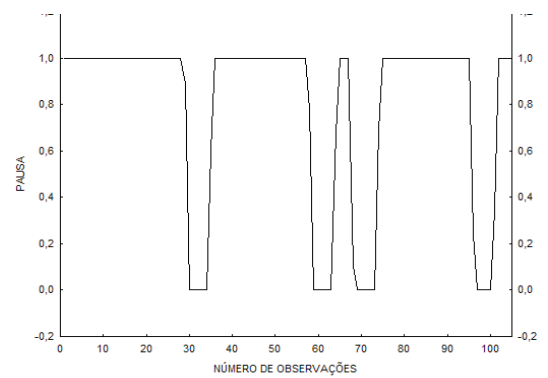

Figura 2: Série temporal de hesitações. Texto com $200 \mathrm{~s}$ de duração, produzido pelo sujeito 1 na descrição de figura. Na ordenada, o valor " 0 " indica a ocorrência de "hesitação", enquanto o valor " 1 " indica o seu contrário. $\mathrm{Na}$ abscissa, cada unidade equivale a $200 \mathrm{~ms}$.

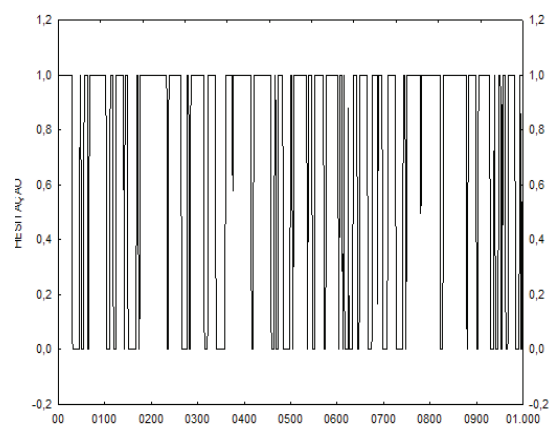

Figura 4: Série temporal de hesitações. Texto com 54 s de duração, produzido pelo sujeito 2 na narrativa autobiográfica. Na ordenada, o valor " 0 " indica a ocorrência de "pausa silenciosa fluente", enquanto o valor " 1 " indica o seu contrário. $\mathrm{Na}$ abscissa, cada unidade equivale a $200 \mathrm{~ms}$.

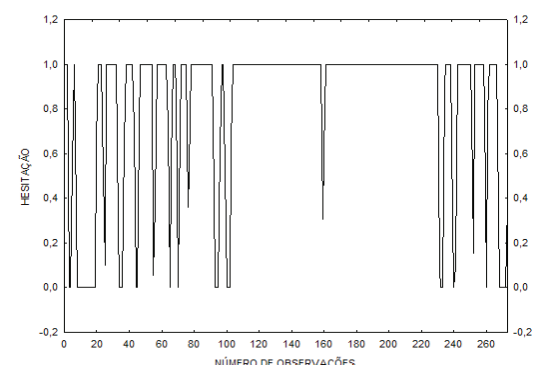


Todos os textos apresentaram, pelo menos, um ciclo periódico de pausa silenciosa e de hesitação $(\mathrm{p}<0,05)$. Este achado sugere que a periodicidade se constitui como um modo de organização desses fenômenos na produção falada. Ou seja, regiões com maior concentração de pausas/hesitações se alternam regularmente com regiões de menor concentração de pausas/hesitações.

Na Tabela 1, estão os dados de estatística descritiva dos ciclos periódicos. A média dos ciclos de pausa se situa em torno de $9 \mathrm{~s}$, enquanto a média dos ciclos de hesitação se situa em torno de $13 \mathrm{~s}$. Esta diferença é significativa $(\mathrm{p}<0,05)$. Além disso, a distribuição dos ciclos de pausa e hesitação é distinta: os ciclos de pausa são mais abundantes abaixo de $6 \mathrm{~s}$, enquanto os de hesitação são mais abundantes acima de $6 \mathrm{~s}$. Portanto, os ciclos de pausas e de hesitações operam em escalas de tempo diferentes. Daí concluímos que pausas e hesitações relacionam-se com níveis diferentes do processamento linguístico-cognitivo.

Tabela 1: Estatística descritiva dos ciclos periódicos de pausas silenciosas fluentes e de hesitações. N $=$ total de textos falados. $\mathrm{n}=$ total de ciclos periódicos nos textos falados. $\mathrm{s}=$ segundos.

\begin{tabular}{lccccccc}
\hline & $\mathrm{N}$ & $\mathrm{n}$ & $\begin{array}{c}\text { Média } \\
(\mathrm{s})\end{array}$ & $\begin{array}{c}\text { Mediana } \\
(\mathrm{s})\end{array}$ & $\begin{array}{c}\text { Mínimo } \\
(\mathrm{s})\end{array}$ & $\begin{array}{c}\text { Máximo } \\
(\mathrm{s})\end{array}$ & $\begin{array}{c}\text { Desvio-padrão } \\
(\mathrm{s})\end{array}$ \\
\hline Pausa & 20 & 39 & 8,72 & 5,85 & 2,04 & 38,80 & 8,59 \\
Hesitação & 20 & 59 & 12,82 & 8,84 & 1,88 & 77,60 & 12,13 \\
\hline
\end{tabular}

Nossos resultados indicam que a emergência de pausas e hesitações é controlada com ciclos de curto, médio e longo prazo, em consonância com achados anteriores (Greene \& Cappella, 1986; Roberts \& Kirsner, 2000; Warner, 1979). Os ciclos de menor período que poderiam ser detectados eram de $400 \mathrm{~ms}$; não encontramos ciclos nesta escala de tempo, diferentemente de Jaffe et al. (1964).

A comparação dos ciclos periódicos de pausa e de hesitação em relação ao tipo textual e à característica autobiográfica dos textos não apresentou nenhum resultado significativo (Tabela 2). A comparação dos ciclos de pausa em relação aos textos autobiográficos e não-autobiográficos foi marginalmente significativa $(\mathrm{p}=0,10)$; assim, é possível que o nível de significância fosse atingido com uma amostra maior. Entretanto, com os resultados atuais, concluímos que os ciclos periódicos são formas robustas de organização da emergência de pausas e hesitações. 
Cadernos de ESTUDOS LINGǘfSTICOS (54.1) - Jan./Jun. 2012

Tabela 2: Comparações entre ciclos periódicos de pausas e hesitações segundo o tipo textual e a característica autobiográfica dos textos.

\begin{tabular}{ccccc}
\hline & $N$ & Média $(s)$ & Desvio-padrão $(s)$ & Teste $t$ \\
\hline CICLOS DE PAUSAS & & & & \\
Descrições de estado & 19 & 8,02 & 8,69 & \\
Narrativas & 20 & 9,38 & 8,67 & $\mathrm{p}=0,99$ \\
Autobiográficos & 21 & 10,84 & 10,57 & \\
Não-autobiográficos & 18 & 6,24 & 4,62 & $\mathrm{p}=0,10$ \\
& & & & \\
CICLOS DE HESITAÇÕES & & & & \\
Descrições de estado & 28 & 14,45 & 14,98 & $\mathrm{p}=0,33$ \\
Narrativas & 31 & 11,34 & 8,81 & \\
Autobiográficos & 32 & 14,06 & 15,21 & $\mathrm{p}=0,40$ \\
Não-autobiográficos & 27 & 11,35 & 6,93 & \\
\hline
\end{tabular}

Em geral, foram encontrados dois ciclos de pausa silenciosa e três de hesitação por texto. Warner (1979) também referiu a presença de mais de um ciclo de pausa silenciosa (fluente e hesitativa) por texto. Deste achado, decorrem duas implicações:

- A primeira explica por que a distribuição periódica das pausas e hesitações geralmente não é aparente. Quando há somente um ciclo de pausa ou hesitação, a periodicidade costuma ser aparente quando os dados são traçados ao longo do tempo: por exemplo, a série temporal de pausa exibida na Figura 1 apresenta uma única frequência de oscilação, com período de $6,07 \mathrm{~s}$. Como geralmente coexistem dois ou mais ciclos de pausa ou hesitação no mesmo texto falado, a distribuição resultante é a somatória desses ciclos, o que faz com que as periodicidades individuais não fiquem tão evidentes. É isso que ocorre nas Figuras 2 e $4 . \mathrm{Na}$ Figura 2, foram encontrados três ciclos de hesitação, com períodos de 22,22, 10,53 e 7,69 s. Na Figura 4, foram encontrados dois ciclos de hesitações, com períodos de 1,94 e 1,88 s. (A Figura 3 será retomada mais adiante).

- A teoria estatística estabelece que cada ciclo periódico possui um mecanismo gerador subjacente (Chatfield, 2004; Morettin \& Toloi, 2004). Assumindo, então, que cada ciclo de pausa e de hesitação é resultado da atividade de um determinado processo linguístico-cognitivo, a presença de mais de um ciclo de pausa ou hesitação por texto sugere que o processamento da linguagem falada ocorre em paralelo na memória operacional. O processamento em paralelo é primordial para a fala fluente (Levelt, 1989).

A impressão de que há regiões com menor ou maior concentração de pausas e hesitações pode ocorrer tanto quando o texto falado apresenta apenas um ciclo periódico ou quando apresenta mais de um ciclo. A série temporal da Figura 3 aparentemente exibe mais pausas na segunda metade do texto. Quando o modelo senoidal do ciclo de 6,93 s é ajustado à série (Figura 5), vemos que as ocorrências de pausa se ajustam aos vales da onda. Na Figura 4, mostramos uma série temporal com aparente não-estacionaridade na região central. Esta série apresentou dois ciclos, um com 1,88 s e outro com 1,94 s (Figura 6). Quando os modelos senoidais de ambos os ciclos são somados (Figura 7), fica claro que a região central com 
MERLO e ALMEIDA BARBOSA - Séries temporais de pausas...

menor quantidade de hesitação foi produzida pela interação entre os ciclos. Portanto, as aparentes não-estacionaridades das séries de pausa e hesitação são devido à interação entre ciclos periódicos.

Figura 5: Ciclo de pausa com período de 6,93 s na narrativa de figura produzida pelo sujeito 3 .

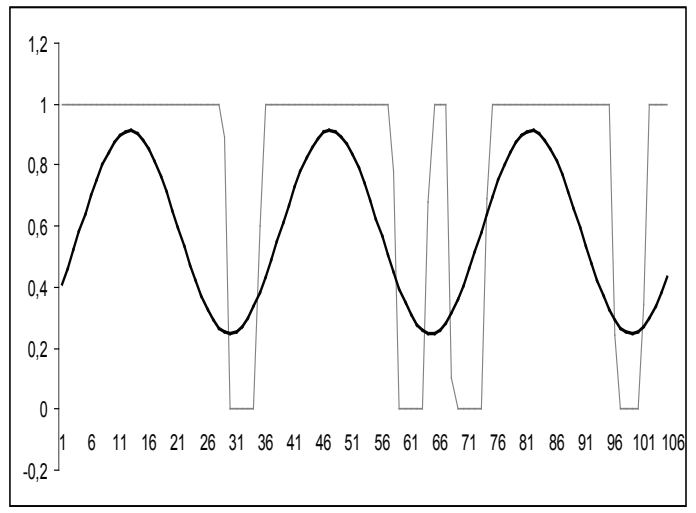

Figura 6: Ciclos de hesitações com períodos de $1,88 \mathrm{~s}$ (onda superior) e $1,94 \mathrm{~s}$ (onda inferior).

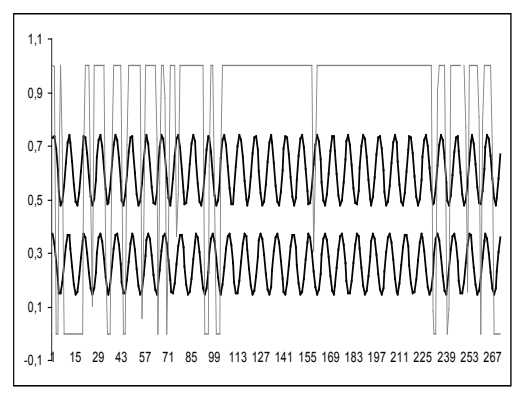

Figura 7: Somatório dos ciclos de hesitações com períodos de 1,88 e 1,94 s. É a interação entre os ciclos que ocasiona a diminuição de hesitações na região central do texto falado.

Uma questão que até então não foi explorada pelos estudos anteriores é o quanto os ciclos periódicos conseguem explicar a variância dos dados. Em média, a variância dos dados explicada pelos ciclos de pausa e hesitação foi $24 \%$ e $29 \%$, respectivamente. A maior parte da variância não pode ser explicada em termos de oscilações periódicas. Assim, o mais correto é dizer que há uma tendência para que pausas e hesitações se distribuam cíclica e periodicamente na fala espontânea. Este resultado indica que o comportamento de pausas e hesitações é estocástico e não determinístico, ou seja, o comportamento desses fenômenos pode ser apenas parcialmente previsto (Chatfield, 2004).

A variância explicada apresentou correlação negativa com a duração dos textos falados (pausas $=-0,60$ e hesitações $=-0,73, p<0,05$ ). Ou seja, quanto menor 
a duração do texto falado, maior a tendência de pausas e hesitações apresentarem distribuição periódica. Duas observações nos parecem relevantes neste aspecto:

- A fala coloquial é geralmente constituída por enunciados curtos, principalmente devido à sua característica dialógica (Linell, 2005). Assim, a maior tendência para a distribuição periódica de pausas e hesitações em textos de menor duração mostra-se compatível com a fala espontânea informal do dia-a-dia.

- O fato de haver menor tendência para a periodicidade conforme a duração textual aumenta não significa que as pausas e hesitações passem a ocorrer aleatoriamente ao longo do tempo. Significa apenas que a distribuição vai deixando de ser cíclica e periódica, mas outro modo de distribuição pode emergir (por exemplo, cíclico e não-periódico).

Outro aspecto ainda não explorado pela literatura da área refere-se à interrelação das séries temporais de pausas e de hesitações.

Uma das medidas que utilizamos, a correlação cruzada defasada, compara se a mudança de estado em uma das séries é acompanhada por mudança de estado na outra série temporal. A média da correlação cruzada defasada foi -2, valor este que é estatisticamente igual a zero $(\mathrm{t}=-1,1, \mathrm{p}=0,29)$. Isso quer dizer que, quando há mudança de estado na série temporal de pausa, imediatamente ocorre mudança de estado na série de hesitação. Assim, pausas e hesitações agem em conjunto na manutenção da fluência.

A outra medida que utilizamos, a análise espectral cruzada, indica se duas séries temporais distintas apresentam ciclos sincronizados. Dos vinte textos falados do corpus, nove apresentaram ciclos de pausa e hesitação com mesmo período: por exemplo, na descrição autobiográfica produzida pelo sujeito 5 (Figura 1), foi encontrado um ciclo de $6,07 \mathrm{~s}$ de pausas e outro ciclo com o mesmo período para hesitações. Oito dos nove ciclos de mesmo período podem ser considerados sincronizados, porque apresentam alta coerência entre si (> 50\%).

Ainda, seis dos oito ciclos sincronizados apresentaram relação de oposição de fase, ou seja, regiões com maior concentração de hesitação coincidiram com regiões com menor concentração de pausa e vice-versa (Figura 8). Apenas dois dos oito ciclos sincronizados apresentaram relação de concordância de fase, ou seja, regiões com maior ocorrência de hesitação e de pausa. Neste último caso, ressaltamos que as ocorrências de hesitação e pausa não são simultâneas, mas se acumulam em torno das mesmas regiões (Figura 9).

Considerando a totalidade de ciclos de pausa e hesitação que foram encontrados no corpus, apenas $16 \%$ deles podem ser considerados sincronizados. Por definição, ciclos sincronizados têm o mesmo período e, portanto, o mesmo mecanismo gerador subjacente (Chatfield, 2004; Morettin \& Toloi, 2004). A pouca ocorrência de ciclos sincronizados sugere que é incomum que pausas e hesitações apresentem funções linguísticas similares. Assim, esta é mais uma evidência que aponta para o fato de pausas e hesitações apresentarem funções psicolinguísticas distintas, embora possa haver sobreposição de funções em certas ocasiões, como indicam as ocorrências de ciclos sincronizados. Através de uma inspeção informal, não encontramos qualquer indício que sugerisse uma razão para a ocorrência de ciclos sincronizados. A presença de ciclos sincronizados 
MERLO e ALMEIDA BARBOSA - Séries temporais de pausas...

não pareceu estar relacionada ao período dos ciclos, à presença de harmônicos, ao número de ciclos por texto, à duração dos textos, à tipologia textual ou à característica autobiográfica do texto.

Figura 8: Ciclos sincronizados de pausas silenciosas fluentes (onda pontilhada) e hesitações (onda contínua). Ciclos em oposição de fase. Período de 6,3 s. Texto com $25 \mathrm{~s}$ de duração, produzido pelo sujeito 3 na descrição de figura.

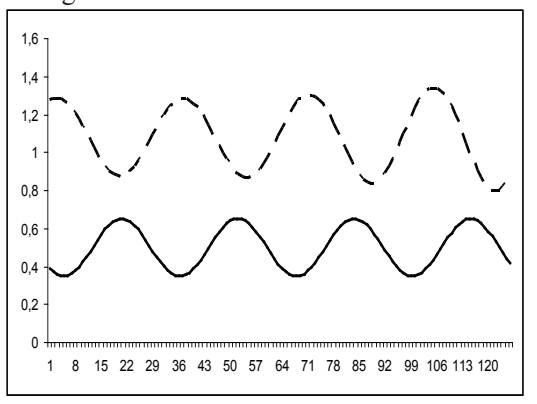

Figura 9: Ciclos sincronizados de pausas silenciosas fluentes (onda pontilhada) e hesitações (onda contínua). Ciclos em concordância de fase. Período de $6,07 \mathrm{~s}$. Texto com $37 \mathrm{~s}$ de duração, produzido pelo sujeito 5 na descrição autobiográfica.

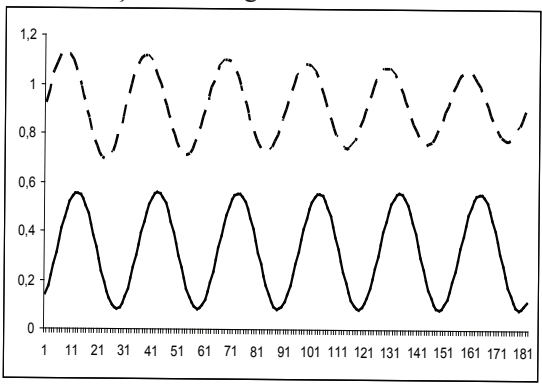

\section{CONCLUSÕES}

O estudo de pausas e hesitações através de séries temporais se mostrou um campo frutífero. Dentre os achados mais importantes do nosso estudo, destacamos:

1. Pausas e hesitações não se aglomeraram no início, no meio ou no final dos textos falados. Elas ocorreram ao longo de toda a extensão dos textos falados.

2. A periodicidade se mostrou um princípio de organização das pausas e hesitações ao longo do tempo, mas este princípio não deve ser entendido como absoluto e, sim, como uma tendência.

3. Pausas e hesitações agiram em conjunto para a manutenção da fluência. Mudanças na ocorrência das pausas causaram imediatamente mudanças na ocorrência das hesitações e vice-versa.

4. Diversos resultados sugeriram que pausas e hesitações apresentam papeis linguísticos distintos: média e distribuição distinta dos ciclos, pouca ocorrência de ciclos sincronizados.

5. Os ciclos de pausa e hesitação não foram sensíveis ao tipo textual e à natureza autobiográfica do texto, desconfirmando, portanto, as quatro hipóteses listadas na Introdução (a, b, c, d). Este achado sugere que os ciclos de pausas e hesitações são manifestações robustas e estáveis durante a produção falada.

\section{REFERÊNCIAS BIBLIOGRÁFICAS}

ADAM, J.-M. \& REVAZ, F. (1997). A análise da narrativa. Lisboa: Gradiva. 
Cadernos de ESTUDOS LINGǘfSTICOS (54.1) - Jan./Jun. 2012

ARAGONÉS, S. (1999). [O pai, o filho e a água do cachorro]. In: Mais do que palavras. São Paulo: Editora Abril. sem página. 1 cartoon: p\&b, 20.5 x $13.5 \mathrm{~cm}$. Material iconográfico.

BARBOSA, P. A. (2006). Incursões em torno do ritmo da fala. Campinas: Pontes, São Paulo: Fapesp.

BOERSMA, P. \& WEENINK, D. (1992-2010). Praat: doing phonetics by computer. Summer Institute of Linguistics.

CAMPIONE, E. \& VÉRONIS, J. (2004). Pauses et hésitations en français spontané. Actes des XXVèmes Journées d'Etude sur la Parole (p. 109-112).

CHATFIELD, C. (2004). The analysis of time series: an introduction. $6^{\text {th }}$ ed. Boca Raton: Chapman \& Hall.

DRITSCHEL, B. H.; WILLIAMS, J. M. G.; BADDELEY, A. D. \& NIMMO-SMITH, I. (1992). Autobiographical fluency: a method for the study of personal memory. Memory \& Cognition, $20(2), 133-140$.

GREENE, J. O. \& CAPPELLA, J. N. (1986). Cognition and talk: the relationship of semantic units to temporal patterns of fluency in spontaneous speech. Language and Speech, 29 (2), 141-157.

JAFFE, J.; CASSOTTA, L. \& FELDSTEIN, S. (1964). Markovian model of time patterns of speech. Science, 144, 884-886.

KIRCHER, T. T. J.; BRAMMER, M. J.; LEVELT, W.; BARTELS, M. \& McGUIRE, P. K. (2004). Pausing for thought: engagement of left temporal cortex during pauses in speech. Neuroimage, $21(1), 84-90$.

KOCH, I. G. V. \& SOUZA E SILVA, M. C. P. (1996). Atividades de composição do texto falado: a elocução formal. In: Castilho, A. T. \& Basilio, M. (orgs). Gramática do português falado. Volume IV: estudos descritivos. Campinas: Editora da UNICAMP e São Paulo: FAPESP. p. 379-410.

LEVELT, W. J. M. (1989). Speaking: from intention to articulation. Cambridge e Londres: The MIT Press and A Bradford Book.

LINELL, P. (2005). The written language bias in Linguistics. Londres e Nova York: Routledge.

MARCUSCHI, L. A. (2006). Hesitação. In: Jubran, C. C. A. S. \& Koch, I. G. V. (org). Gramática do português culto falado no Brasil: construção do texto falado (p. 48-70). Vol 1. Editora da Unicamp, Campinas.

MORAES, E. (2003). As horas. 1 figura: p\&b, 21 x $29.7 \mathrm{~cm}$. Material iconográfico.

MORETTIN, P. A. \& TOLOI, C. M. C. (2004). Análise de séries temporais. São Paulo: Ed. Edgard Blücher.

O'CONNELL, D. C. \& KOWAL, S. (2004). The history of research on the filled pause as evidence of The Written Language Bias in Linguistics (Linell, 1982). Journal of Psycholinguistic Research, 33 (6), 459-474.

ROBERTS, B. \& KIRSNER, K. (2000). Temporal cycles in speech production. Language and Cognitive Processes, 15 (2), 129-157.

SCARPA, E. M. (2006). (Ainda) sobre o sujeito fluente. In: Lier-DeVitto, M. F. \& Arantes, L. (org). Aquisição, Patologias e Clínica de Linguagem. São Paulo: Educ (PUC-SP). p. 161-180. 
MERLO e ALMEIDA BARBOSA - Séries temporais de pausas... (1995). Sobre o sujeito fluente. Cadernos de Estudos Lingüísticos, 29, 163-184.

SCHACHTER, S.; CHRISTENFELD, N.; RAVINA, B. \& BILOUS, F. (1991). Speech disfluency and the structure of knowledge. Journal of Personality and Social Psychology, 60 (3), 362-367.

SOUZA E SILVA, M. C. P. \& KOCH, I. G. V. (2002). Estratégias de desaceleração do texto falado. In: Kato, M. A. (org). Gramática do português falado. Volume V: convergências. $2^{\mathrm{a}}$ ed. Campinas: Editora da UNICAMP. p. 329-340.

WARNER, R. M. (1998). Spectral analysis of time-series data. Nova York e Londres: The Guilford Press. (1979). Periodic rhythms in conversational speech. Language and Speech, 22 (4), 381-396.

WINGATE, M. E. (1987). Fluency and disfluency; illusion and identification. Journal of Fluency Disorders, 12, 79-101. 